\title{
openheart Genetic, clinical and pharmacological determinants of out-of-hospital cardiac arrest: rationale and outline of the AmsteRdam Resuscitation Studies (ARREST) registry
}

\author{
M T Blom, ${ }^{1}$ D A van Hoeijen, ${ }^{1}$ A Bardai, ${ }^{1,2}$ J Berdowski, ${ }^{1}$ P C Souverein, ${ }^{3}$ \\ M L De Bruin, ${ }^{3}$ R W Koster, ${ }^{1}$ A de Boer, ${ }^{3} \mathrm{H} \mathrm{L} \mathrm{Tan}{ }^{1}$
}

To cite: Blom MT, van Hoeijen DA, Bardai A, et al. Genetic, clinical and pharmacological determinants of out-ofhospital cardiac arrest: rationale and outline of the AmsteRdam Resuscitation Studies (ARREST) registry. Open Heart 2014;1:e000112. doi:10.1136/openhrt-2014000112

Received 5 March 2014 Revised 6 June 2014 Accepted 15 July 2014

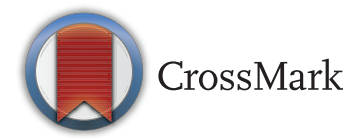

${ }^{1}$ Department of Cardiology, Academic Medical Center, University of Amsterdam, Amsterdam, The Netherlands ${ }^{2}$ Interuniversity Cardiology Institute Netherlands, Utrecht, The Netherlands

${ }^{3}$ Division of

Pharmacoepidemiology and Clinical Pharmacology, Utrecht Institute for Pharmaceutical Sciences, Utrecht, The Netherlands

Correspondence to Dr Hanno L Tan; h.l.tan@amc.nl

\section{ABSTRACT}

Introduction: Out-of-hospital cardiac arrest (OHCA) is a major public health problem. Recognising the complexity of the underlying causes of OHCA in the community, we aimed to establish the clinical, pharmacological, environmental and genetic factors and their interactions that may cause OHCA.

Methods and analysis: We set up a large-scale prospective community-based registry (AmsteRdam Resuscitation Studies, ARREST) in which we prospectively include all resuscitation attempts from OHCA in a large study region in the Netherlands in collaboration with Emergency Medical Services. Of all OHCA victims since June 2005, we prospectively collect medical history (through hospital and general practitioner), and current and previous medication use (through community pharmacy). In addition, we include DNA samples from OHCA victims with documented ventricular tachycardia/fibrillation during the resuscitation attempt since July 2007 . Various study designs are employed to analyse the data of the ARREST registry, including case-control, cohort, case only and case-cross over designs.

Ethics and dissemination: We describe the rationale, outline and potential results of the ARREST registry. The design allows for a stable and reliable collection of multiple determinants of OHCA, while assuring that the patient, lay-caregiver or medical professional is not hindered in any way. Such comprehensive data collection is required to unravel the complex basis of OHCA. Results will be published in peer-reviewed journals and presented at relevant scientific symposia.

\section{INTRODUCTION}

Sudden cardiac arrest is a major health problem in the affluent world, accounting for $15-20 \%$ of all natural deaths in adults in the USA and Western Europe, and for up to $50 \%$ of all cardiovascular deaths. ${ }^{1}$ In the vast majority, it occurs in the community

\section{KEY MESSAGES}

What is already known about this subject?

- Out-of-hospital cardiac arrest (OHCA) in the community is a multifactorial phenomenon highly heterogeneous causes. Resolving these causes has great clinical relevance, because OHCA accounts for $20 \%$ of mortality in industrialized countries. These efforts have so far been strongly hampered by the fact that, while it requires collection of sufficient numbers of deeply annotated (phenotype, genotype) OHCA cases, such cases have been extremely hard to obtain as case ascertainment requires timely identification of $\mathrm{OHCA}$ occurrences.

What does this study add?

- ARREST is a prospective registry specifically designed to obtain an unbiased collection of cases of all-cause OHCA with ECG-documented VTNF in the community. ARREST is aimed at identifying genetic, clinical, pharmacological and environmental determinants of OHCA in the community.

How might this impact on clinical practice?

- Thanks to the collection of large numbers of deep-phenotyped OHCA cases with DNA information, made possible by the ARREST infrastructure, studies into the complex etiology of OHCA are possible. Recognizing the complexity of the underlying causes and risk factors of $\mathrm{OHCA}$ in the community may enhance the development of therapies to prevent $\mathrm{OHCA}$.

(out-of-hospital cardiac arrest, OHCA). It is often (up to $50 \%$ ) the first sign of heart disease. ${ }^{2}{ }^{3}$ Survival rates are increasing, but remain low despite intense efforts at improving treatment of OHCA victims. ${ }^{4-6}$ Prevention of OHCA is clearly desirable and requires elucidation of the underlying disease processes and risk factors. OHCA is 
usually caused by cardiac arrhythmias, that is, ventricular tachycardia/ventricular fibrillation (VT/VF). VT/VF may stem from multiple mechanisms, ultimately resulting in disrupted cardiac electrophysiology. On the level of an individual patient, multiple conditions, inherited and acquired, interact to cause these disruptions and result in $\mathrm{VT} / \mathrm{VF}^{3}$

Important predictors of OHCA, including heart failure, cardiac ischaemia and diabetes, have previously been identified. However, these risk factors are often unrecognised prior to OHCA and are thus predictive in only a minority of OHCA victims. Furthermore, additional non-cardiac risk factors for OHCA (eg, depression, epilepsy, environmental factors) have been proposed, suggesting that OHCA in the community is multifactorial. ${ }^{7-10}$ Moreover, various drugs have been associated with OHCA, ultimately leading to their withdrawal from the market. ${ }^{11}{ }^{12}$ Apparently, the strict premarketing safety screening systems lack the ability to identify all drugs that increase the risk of OHCA. Of note, this also applies to non-cardiac drugs, that is, drugs prescribed for non-cardiac disease. Such drugs may cause VT/VF because they impact on cardiac electrophysiology by modulating cardiac ion channel function. Importantly, VT/VF risk from drug use often requires the added presence of other risk factors. For instance, VT/VF risk conferred by (cardiac or noncardiac) sodium channel blocking drugs is particularly high in patients with heart failure and/or cardiac ischaemia. ${ }^{13}{ }^{14}$ Genetic factors may also modulate VT/VF risk. For instance, observational studies have indicated that sudden death of a family member is a risk factor for OHCA. ${ }^{15-17}$ Genetic markers for increased OHCA risk in the community have started to be found, ${ }^{18}{ }^{19}$ but the role of causative genes with large effect sizes remains to be established.

Recognising the complexity of the underlying causes of OHCA in the community, we aimed to uncover the clinical, pharmacological, environmental and genetic factors, and their interactions that may cause OHCA. To this end, we setup a large-scale community-based registry (AmsteRdam Resuscitation Studies, ARREST) in which we prospectively include all resuscitation attempts from OHCA in a large study region in the Netherlands. Of all OHCA victims, we collect medical history, current and previous medication use, and DNA samples. The objective of this paper is to describe the design, rationale and outline of this registry, hereafter referred to as the ARREST registry.

\section{METHODS}

\section{Design and setting}

ARREST is an ongoing, prospective observational registry of all OHCAs in the study region North Holland, a province of The Netherlands. ARREST was setup in June 2005 to establish the determinants of outcome of OHCA. ${ }^{20-22}$ In July 2007, its aims were extended to include insight into the genetic, clinical, pharmacological and environmental determinants of OHCA in the community. ${ }^{10} 1819$ To minimise selection bias, data of all resuscitation attempts with involvement of emergency medical services (EMS) in the study region are collected and stored in the ARREST database. The study region covers $2404 \mathrm{~km}^{2}$ (urban and rural communities) and has a population of 2.4 million people. All studies with the ARREST registry are conducted according to the principles expressed in the Declaration of Helsinki. Since OHCA is an emergency situation, consent cannot be asked before data collection. From all participants who survive OHCA, written informed consent for collection and use of medical and pharmacological information and DNA samples is obtained after discharge from the hospital. The Ethics Committee of the Academic Medical Center, a teaching hospital in Amsterdam, approved the study protocol, and the use of data from patients who did not survive OHCA and of data needed to evaluate the outcome of the resuscitation attempt.

\section{EMS system in the study region}

In a medical emergency, people dial the national emergency number. When the EMS dispatcher suspects OHCA, he/she dispatches two ambulances. Ambulance personnel are equipped with a manual defibrillator and qualified to perform Advanced Life Support. In part of the study area, the EMS dispatcher also sends out first responders (firefighters or police officers) equipped with an automated external defibrillator (AED) in the event of a suspected OHCA. In addition, the placements of AEDs in public areas has been facilitated by public and private initiatives during the study period, but is not centrally controlled or directed. ${ }^{20}$ All four EMS services in the study region participate in ARREST.

\section{Data collection}

\section{Resuscitation parameters}

After each resuscitation attempt, EMS paramedics routinely send the continuous ECG and impedance recordings from their manual defibrillators to the study centre by modem. When an AED is used prior to EMS arrival, a member of the ARREST study personnel visits the AED site shortly after the OHCA, and collects the AED ECG recording. All ECGs are stored and analysed with dedicated software (Code Stat Reviewer 7.0, Physio Control, Redmond, Washington, USA), allowing for analysis of heart rhythm/arrhythmia during the resuscitation attempt. Data items concerning the resuscitation procedure are collected according to the Utstein recommendations. ${ }^{23}$ Ambulance personnel are obliged by protocol to call the study centre after every OHCA to provide additional information on the resuscitation (eg, whether OHCA was witnessed, whether basic life support was provided before arrival of ambulance personnel, whether the patient died at the resuscitation site or was transported to a hospital). Hospital files are reviewed by ARREST study personnel to retrieve data of inhospital 
resuscitation care/treatment (therapeutic hypothermia, angiography), outcome and neurological status when discharged alive.

OHCA is defined as the out-of-hospital occurrence of cessation of cardiac mechanical activity as confirmed by the absence of signs of circulation. ${ }^{23}$ All arrests are deemed to result from cardiac causes unless an unequivocal non-cardiac cause (ie, trauma, drowning, intoxication) is documented. All EMS and hospital case files are reviewed to verify the presence or absence of a non-cardiac cause. In addition, data items concerning medication use and medical history are collected. For the sake of DNA discovery studies, the best possible ascertainment of a cardiac cause of OHCA is achieved by collecting DNA samples only of patients with documented VT/VF at any time during resuscitation. Patients in whom only asystole (but no VT/VF) is recorded, are excluded, as asystole is the end stage of any cardiac arrest and may be due to non-cardiac causes. ${ }^{24}$

\section{DNA samples}

In order to avoid interventions during the resuscitation attempt for research purposes only, DNA samples are collected as follows. Of patients who die on the scene and are not transported to the hospital, DNA is extracted from the endotracheal tube placed for ventilation during the resuscitation attempt (no blood is usually drawn before hospital admission). Endotracheal tubes are collected at ambulance posts or emergency rooms by study personnel. Of patients who reach the hospital alive, DNA is extracted from blood lymphocytes of blood that is routinely collected and analysed in the hospital's laboratory for patient care. Unused blood (available in $>95 \%$ of the patients) is sent to the ARREST study centre or collected by study personnel, and is used for DNA analysis and to determine metabolic parameters.

If future analyses of the collected DNA material reveal pathogenic mutations that require medical action and if the patient has indicated that he/she wishes to be informed when giving informed consent, the patient is contacted through his/her general practitioner (GP). Genetic counselling is offered to the patient and the patient's relatives in such a case.

\section{Medical information}

Medical information (medical history including mental health, current disease diagnosis, survival status and, if available, postmortem examination) is collected by contacting the hospital of admission and the patient's physician (GP), and conducting telephone interviews with surviving patients. In the Netherlands, every citizen has a GP who acts as a gatekeeper for all medical care. GP records contain information on diagnoses from GPs/ specialists that are based on guidelines for GPs/specialists. Diagnoses in the GP files are recorded according to the International Classification of Primary Care system. The GPs are asked to mark in a questionnaire whether their patient was diagnosed prior to their OHCA with any medical condition. In addition, GPs are asked to send all medical correspondence with all treating medical specialists to the ARREST study centre.

\section{Pharmacological information}

Information regarding medication use (current medication at the time of OHCA and in the year before OHCA) is obtained by contacting the patient's community pharmacist. Exposure to medication is determined by reviewing dispensing information prior to the date of OHCA. Dosage and duration of medication use is recorded of all medication use and used to calculate the time during which a patient is exposed.

\section{Environmental information}

To assess the influence of environmental factors on OHCA risk, we analyse the socioeconomic and environmental characteristics of the patient's place of residency. Such data on a neighbourhood level are available from Statistics Netherlands, a Dutch governmental institution.

\section{Data analysis}

Data and DNA material collected by the ARREST registry can be analysed using various study designs.

We employ case-control designs, collaborating with registries and studies that provide suitable controls for ARREST OHCA-cases. Cases are matched to controls according to age, sex and OHCA date. Controls are drawn from a registry that contains drug dispensing records from community pharmacies (PHARMO Institute for Drug Outcomes Research, Utrecht, the Netherlands http://www.pharmo.nl/), ${ }^{10} 25$ and from a database that contains the complete medical records of $>60000$ people from a large group of GPs in the study area (HAG-net-AMC). ${ }^{9}{ }^{26}$ Genetic data of ARREST OHCA-cases are compared to data registries with DNA samples of normal control populations. ${ }^{18} 19$

In a cohort study, we compare a group of ARREST OHCA cases with a particular property to a group of cases without that property. For example, we study ARREST OHCA cases with and without obstructive pulmonary disease, and compare their survival to hospital discharge. ${ }^{22}$

Case only designs are employed when studying effects of interactions on OHCA risk, for example, between a particular gene profile and exposure to study drugs; in this way, we test the hypothesis that certain gene profiles may modify the risk of the study drug on OHCA. ${ }^{27}$

In a case cross-over design, properties of the ARREST OHCA case (eg, exposure to study drug) are compared with the same property at an earlier time point (eg, 6 months before OHCA), thereby reducing confounding and selection bias.

\section{RESULTS}

Data collection of resuscitation parameters started in June 2005. In July 2007, we started collecting DNA 


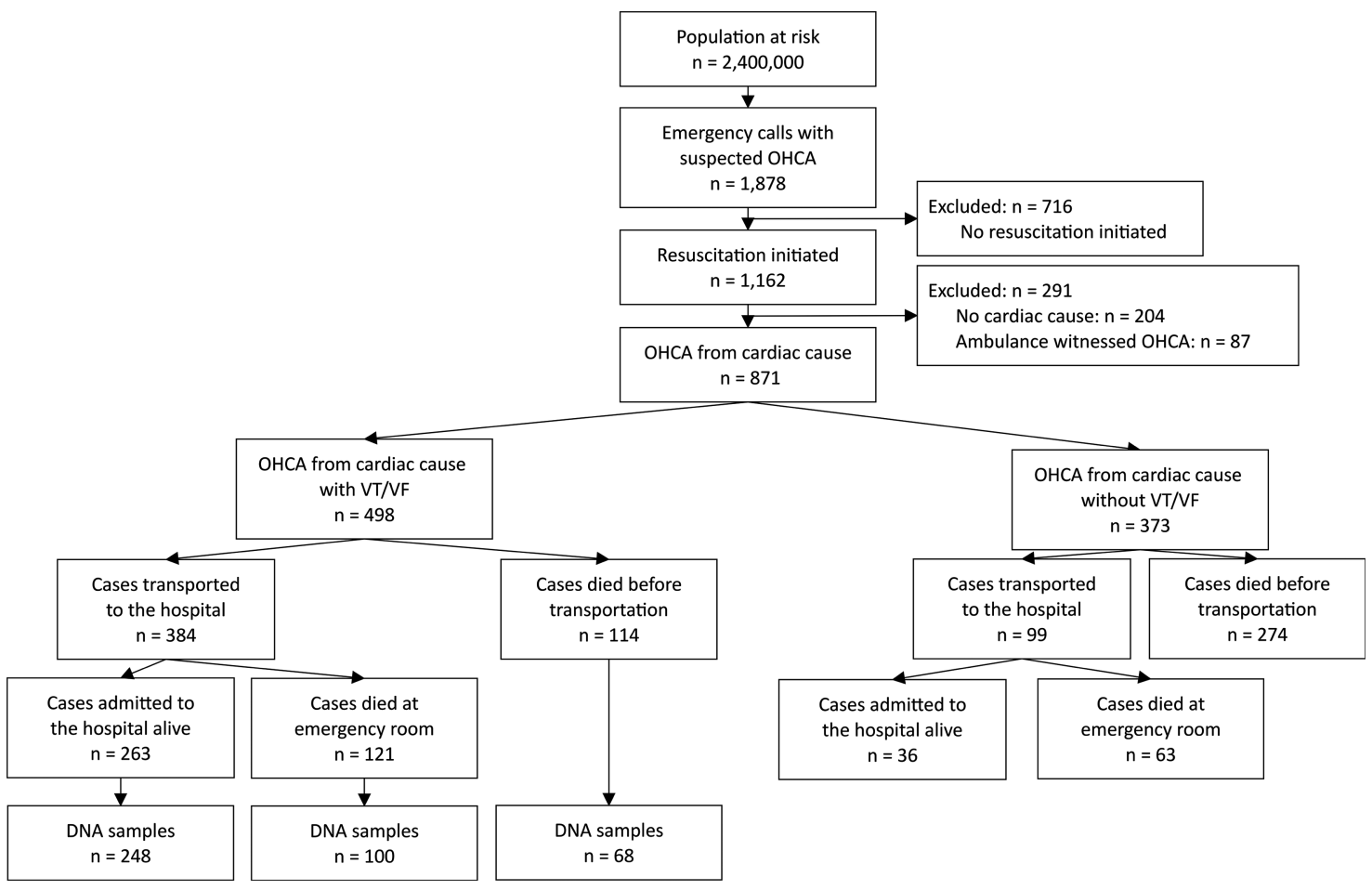

Figure 1 Patient inclusion in the year 2009 (OHCA, out-of-hospital cardiac arrest; VT/VF, ventricular tachycardia/ventricular fibrillation).

samples. Each year, we collect data from $\sim 1000$ resuscitation attempts, $\sim 50 \%$ of which have VT/VF documentation. Figure 1 shows the inclusion scheme of all VT/VF cases that we have included in the year 2009, the first year in which all hospitals and ambulance services participated in DNA collection. In this 1-year period, we registered 1162 resuscitation attempts, of which 871 had a cardiac cause without EMS witness. In 498 cases, VT/ $\mathrm{VF}$ was documented. Of these VT/VF cases, 23\% (114) died on the scene, $24 \%$ (121) died in the emergency room before hospital admission, while 53\% (263) were admitted to the hospital. DNA samples were collected in $60 \%$ of cases who died on the scene, in $83 \%$ of cases who died in the emergency room and in $94 \%$ of cases who were admitted to the hospital.

Table 1 presents medication data per indication category of ARREST OHCA cases $(\mathrm{N}=1787$, mean age $66.5,77 \%$ male) collected in the period 2005-2009, and age/sex/ OHCA date-matched non-OHCA controls from the PHARMO database $(\mathrm{N}=7698)$. As expected, medication

Table 1 Medication use (ATC classification system categories) of OHCA cases and age-matched/sex-matched controls

$\mathrm{N}$

Male

Mean age (SD), years

A-Alimentary tract and metabolism

B-Blood and blood forming organs

C-Cardiovascular system

G-Genito urinary system and sex hormones

$\mathrm{H}$-Systemic hormonal preparations, excluding sex hormones

J-Anti-infectives for systemic use

$\mathrm{L}$-Antineoplastic and immunomodulating agents

M-Musculoskeletal system

$\mathrm{N}$-Nervous system

$\mathrm{P}$-Antiparasitic products, insecticides and repellents

R-Respiratory system

\section{ARREST OHCA with documented VT/VF}

1787
$1376(77.0)$
$66.5(13.7)$
$810(45.3)$
$925(51.8)$
$1268(71.0)$
$221(12.4)$
$181(10.1)$
$410(22.9)$
$53(3.0)$
$354(19.8)$
$557(31.2)$
$12(0.7)$
$426(23.8)$

PHARMO controls

7698

$5939(77.1)$

$66.4(13.8)$

$2607(33.9)$

$2401(31.2)$

$3903(50.7)$

$911(11.8)$

$563(7.3)$

$1416(18.4)$

$177(2.3)$

$1420(18.4)$

$1966(25.5)$

$73(0.9)$

$1524(19.8)$

Data are $\mathrm{N}(\%)$, unless otherwise indicated.

ATC, Anatomical Therapeutic Chemical Classification System; OHCA, out-of-hospital cardiac arrest; VF, ventricular fibrillation; VT, ventricular tachycardia. 
DNA sample inclusion until January $2014(\mathrm{~N}=3005)$

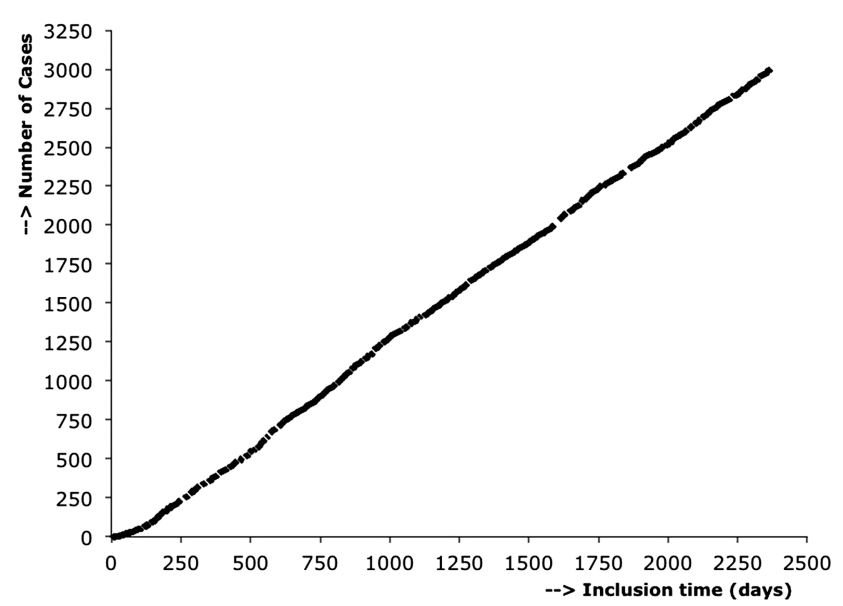

Figure 2 Collection of DNA samples of out-of-hospital cardiac arrest victims with VT/VF (ventricular tachycardia/ ventricular fibrillation) July 2007-January 2014.

use for cardiovascular diseases was higher among cases than controls. Drugs used for several other categories were also more prevalent among cases, inviting further research.

Figure 2 shows the inclusion rate of DNA samples in the period July 2007-January 2014. We included 3005 cases and continue to collect DNA samples at the same rate. The average yield of DNA from blood samples was $160 \mu \mathrm{g}$ DNA, while tubes yielded $6.9 \mu \mathrm{g}$ DNA. Inclusion rate was stable over the past years.

\section{DISCUSSION}

The pathophysiology of OHCA is extremely complex, and its causes are highly heterogeneous. In many patients, it is not possible to single out one individual cause. Instead, cardiac arrest results from various interacting causes and circumstances and a final trigger. This complicates community-based research into the aetiology of OHCA to an enormous extent. Furthermore, since OHCA occurs in such an unpredictable manner, it is difficult to obtain consent, precise data on circumstances of OHCA and, most notably, DNA samples. To increase the chances for discovery of relevant DNA profiles, ascertainment of a cardiac cause of OHCA is crucial. To this end, we chose to define a clear and unambiguous selection criterion: documented VT/VF. We expect that with this precise case definition, we will create a more homogeneous cohort of OHCA cases for analysis, thereby increasing our ability to identify genetic risk factors. Our close collaboration with EMS services in the study region enables us to include all OHCA cases in the study region, thereby enabling us to also compare patients with or without VT/VF. Our study design thus allows us to study the full range of clinical, pharmacological, environmental and genetic risk factors of OHCA, and their interactions.
Of added importance, our study group has the ability to complement findings from these association studies with mechanistic studies in the experimental laboratory. This laboratory is equipped with a comprehensive range of experimental techniques, including molecular biology, molecular genetics, single-cell electrophysiology (patch-clamp studies), and tissue, organ and in vivo electrophysiology. Moreover, our study group is also embedded in the cardiology and cardiogenetics departments. This organisation in which these various domains are interwoven offers the possibility that research questions from one domain feed into the other domains. This allows for a multifaceted approach to resolve the causes of OHCA in the community. ${ }^{28}$

Finally, the ARREST infrastructure is suitable to study the causes of OHCA as well as its outcome. The fact that data regarding the resuscitation attempt are collected according to Utstein recommendations enables us to study survival at the various stages of postresuscitation care. This allows for evaluation of the effects of various interventions in the chain-of-survival care after OHCA.

\section{CONCLUSION}

We describe the rationale, outline and potential results of the ARREST database. To our knowledge, ARREST is the first prospective population-based registry of patients with OHCA aimed at identifying genetic, clinical, pharmacological and environmental determinants of OHCA with documented VT/VF. The data presented show that our study design allows for a stable and reliable registry of OHCA.

Contributors MTB, AB, JB, PCS, RWK, AdB and HLT conceived the study. RWK and HLT obtained funding for the study and supervised it. MTB, DAvH, $A B$ and JB collected the data. MTB, DAvH, AB, JB and HLT wrote the manuscript. PCS, MLDB, RWK and AdB provided critical revisions of the manuscript.

Funding The ARREST registry is supported by a grant from the Netherlands Heart Foundation, Den Haag, The Netherlands (grant 2006-B179), and by an unconditional grant from Physio Control Inc, Redmond, WA. The ARREST registry is further supported by The Netherlands Organisation for Scientific Research (NWO, grant ZonMW Vici 918.86.616 to dr Tan), the Dutch Medicines Evaluation Board (MEB/CBG), the European Community's Seventh Framework Programme (FP7, grant 241679, ARITM0) and Biobanking and Biomolecular Research Infrastructure, The Netherlands (BBMRI-NL).

\section{Competing interests None.}

Ethics approval The Ethics Committee of the Academic Medical Center, Amsterdam, The Netherlands.

Provenance and peer review Not commissioned; externally peer reviewed.

Data sharing statement No additional data are available.

Open Access This is an Open Access article distributed in accordance with the Creative Commons Attribution Non Commercial (CC BY-NC 4.0) license, which permits others to distribute, remix, adapt, build upon this work noncommercially, and license their derivative works on different terms, provided the original work is properly cited and the use is non-commercial. See: http:// creativecommons.org/licenses/by-nc/4.0/ 


\section{REFERENCES}

1. Myerburg RJ, Castellanos A. Cardiac arrest and sudden cardiac death. In: Libby P, Bonow RO, Mann DL, Zipes DP. eds. Braunwald's heart disease: a textbook of cardiovascular medicine. Oxford, UK: Elsevier, 2007:933-74.

2. Rea TD, Crouthamel M, Eisenberg MS, et al. Temporal patterns in long-term survival after resuscitation from out-of-hospital cardiac arrest. Circulation 2003;108:1196-201.

3. Huikuri HV, Castellanos A, Myerburg RJ. Sudden death due to cardiac arrhythmias. N Engl J Med 2001;345:1473-82.

4. Iwami T, Nichol G, Hiraide A, et al. Continuous improvements in "chain of survival" increased survival after out-of-hospital cardiac arrests: a large-scale population-based study. Circulation 2009;119:728-34.

5. Berdowski J, Berg RA, Tijssen JG, et al. Global incidences of out-of-hospital cardiac arrest and survival rates: systematic review of 67 prospective studies. Resuscitation 2010;81:1479-87.

6. Aufderheide TP, Yannopoulos D, Lick CJ, et al. Implementing the 2005 American Heart Association Guidelines improves outcomes after out-of-hospital cardiac arrest. Heart Rhythm 2010;7:1357-62.

7. Empana JP, Jouven X, Lemaitre RN, et al. Clinical depression and risk of out-of-hospital cardiac arrest. Arch Intern Med 2006;166:195-200.

8. Silverman RA, Ito K, Freese J, et al. Association of ambient fine particles with out-of-hospital cardiac arrests in New York City. Am J Epidemiol 2010;172:917-23.

9. Bardai A, Lamberts RJ, Blom MT, et al. Epilepsy is a risk factor for sudden cardiac arrest in the general population. PLOS ONE 2012;7: e42749

10. Warnier MJ, Blom MT, Bardai A, et al. Increased risk of sudden cardiac arrest in obstructive pulmonary disease: a case-control study. PLoS ONE 2013;8:e65638.

11. Ray WA, Chung CP, Murray KT, et al. Atypical antipsychotic drugs and the risk of sudden cardiac death. N Engl J Med2009;360:225-35.

12. Qureshi ZP, Seoane-Vazquez E, Rodriguez-Monguio R, et al. Market withdrawal of new molecular entities approved in the United States from 1980 to 2009. Pharmacoepidemiol Drug Saf 2011;20:772-7.

13. Greenberg HM, Dwyer EM Jr, Hochman JS, et al. Interaction of ischaemia and encainide/flecainide treatment: a proposed mechanism for the increased mortality in CAST I. Br Heart $J$ 1995;74:631-5.

14. Shaw RM, Rudy Y. Electrophysiologic effects of acute myocardial ischemia: a theoretical study of altered cell excitability and action potential duration. Cardiovasc Res 1997;35:256-72.

15. Friedlander $Y$, Siscovick DS, Weinmann S, et al. Family history as a risk factor for primary cardiac arrest. Circulation 1998;97:155-60.

16. Jouven X, Desnos M, Guerot C, et al. Predicting sudden death in the population: the Paris Prospective Study I. Circulation 1999;99:1978-83.
17. Dekker LR, Bezzina CR, Henriques JP, et al. Familial sudden death is an important risk factor for primary ventricular fibrillation: a case-control study in acute myocardial infarction patients. Circulation 2006;114:1140-5.

18. Bezzina CR, Pazoki R, Bardai A, et al. Genome-wide association study identifies a susceptibility locus at 21q21 for ventricular fibrillation in acute myocardial infarction. Nat Genet 2010;42:688-91.

19. Arking DE, Junttila MJ, Goyette $P$, et al. Identification of a sudden cardiac death susceptibility locus at 2q24.2 through genome-wide association in European ancestry individuals. PLOS Genet 2011;7: e1002158.

20. Berdowski J, Blom MT, Bardai A, et al. Impact of onsite or dispatched automated external defibrillator use on survival after out-of-hospital cardiac arrest. Circulation 2011;124:2225-32.

21. Bardai A, Berdowski J, van der Werf C, et al. Incidence, causes, and outcomes of out-of-hospital cardiac arrest in children. A comprehensive, prospective, population-based study in the Netherlands. J Am Coll Cardiol 2011;57:1822-8.

22. Blom MT, Warnier MJ, Bardai A, et al. Reduced in-hospital survival rates of out-of-hospital cardiac arrest victims with obstructive pulmonary disease. Resuscitation 2013;84:569-74.

23. Jacobs I, Nadkarni V, Bahr J, et al. Cardiac arrest and cardiopulmonary resuscitation outcome reports: update and simplification of the Utstein templates for resuscitation registries: a statement for healthcare professionals from a task force of the International Liaison Committee on Resuscitation (American Heart Association, European Resuscitation Council, Australian Resuscitation Council, New Zealand Resuscitation Council, Heart and Stroke Foundation of Canada, Inter-American Heart Foundation, Resuscitation Council of Southern Africa). Circulation 2004;110: 3385-97.

24. Engdahl J, Bang A, Lindqvist J, et al. Can we define patients with no and those with some chance of survival when found in asystole out of hospital? Am J Cardiol 2000;86:610-14.

25. Herings RMC. Pharmo, a record linkage system for post marketing surveillance of prescription drugs in the Netherlands [Thesis Department of Pharmacoepidemiology and Pharmacotherapy Utrecht]. The Netherlands: Utrecht University, 1993.

26. van Doormaal FF, Atalay S, Brouwer HJ, et al. Idiopathic superficial thrombophlebitis and the incidence of cancer in primary care patients. Ann Fam Med 2010;8:47-50.

27. Khoury MJ, Flanders WD. Nontraditional epidemiologic approaches in the analysis of gene-environment interaction: case-control studies with no controls!. Am J Epidemiol 1996;144:207-13. Review.

28. Bardai A, Amin AS, Blom MT, et al. Sudden cardiac arrest associated with use of a non-cardiac drug that reduces cardiac excitability: evidence from bench, bedside, and community. Eur Heart J 2013;34:1506-16. 